\title{
Prevalencia y significado clínico de los cánceres de próstata focales e incidentales
}

\author{
Lujan Galán M, García Tello A, Pascual Mateo C, Llanes González L, García Mediero JM, \\ Ángulo Cuesta J, Berenguer Sánchez A.
}

Hospital Universitario de Getafe, Servicio de Urología, Madrid.

Actas Urol Esp. 2007;31(8):819-824

\section{RESUMEN}

PREVALENCIA Y SIGNIFICADO CLINICO DE LOS CANCERES DE PROSTATA FOCALES E INCIDENTALES

Objetivos: Calcular la proporción de cánceres de próstata (CaP) focales e incidentales en nuestro ámbito, investigar su relación con los hallazgos en la pieza de prostatectomía radical (PR), así como establecer su relevancia clínica en términos de progresión y supervivencia.

Material y Métodos: Hemos seleccionado los pacientes con cáncer focal, definiendo éste como un máximo de $3 \mathrm{~mm}$ en uno o dos cilindros adyacentes en la biopsia prostática (dirigidas mediante ecografia transrectal, de modo randomizado sextante). Adicionalmente, hemos incluido un grupo de pacientes con cáncer incidental Tla (diagnosticados tras prostatectomía, no palpables, con tumor en menos del $5 \%$ del tejido obtenido). Se ha calculado la proporción de estos cánceres en nuestra área sanitaria sobre el total de tumores detectados, y registrando las características clínicas de dichos tumores (edad, PSA, grado y score de Gleason), así como el tratamiento efectuado. En los casos sometidos a PR, se han analizado los parámetros patológicos de la pieza. Por último, se ha realizado un análisis de supervivencia (Kaplan-Meier) para describir la evolución de estos pacientes en cuanto al tiempo hasta la progresión de la enfermedad y al fallecimiento por CaP.

Resultados: De 819 pacientes con CaP, $46(5,6 \%)$ presentaron cáncer focal y $23(2,8 \%)$ tumores estadio Tla. Ninguno de los pacientes con tumor incidental (Tla) fue sometido a PR frente a 17 de los 46 cánceres focales T1c (37\%). Aunque ninguno de los casos mostró enfermedad extracapsular, invasión de vesículas seminales, o invasión ganglionar, se detectó enfermedad relevante (estadio pT2b o superior) en 15 casos $(88,2 \%)$ y un score de Gleason patológico $>7$ en 2 casos $(2,9 \%)$.

Con una media de seguimiento de 37,6 meses (error estándar 4,26), la probabilidad de permanecer libre de cualquier tipo de progresión para los Tla a los 2 y 5 años fue de 75,4\% y 57,1\% respectivamente, siendo de 94,4\% y $94,4 \%$ respectivamente para los Tlc. No se registraron fallecimientos por CaP en esta serie durante el seguimiento mencionado.

Conclusión: En nuestra experiencia, el hallazgo de cáncer microscópico o focal en la biopsia randomizada sextante se corresponde una elevada proporción de tumores clínicamente relevantes en la pieza de PR (88\%). Creemos que la actitud expectante ante dichos hallazgos en la biopsia prostática debe ser sometida a crítica.

Palabras clave: Biopsia prostática. Cáncer de próstata. Prostatectomía radical.

\section{ABSTRACT}

PREVALENCE AND CLINICAL MEANING OF FOCAL AND INCIDENTAL PROSTATE CANCERS

Objectives: To calculate the proportion of focal and incidental prostate cancers (PCa) in our setting, to study their relationship with the findings in radical prostatectomy (RP) specimens, and to establish their clinical relevance in terms of progression and survival.

Material and methods: We selected patients with focal cancer, defined as a maximum extent of 3 mm in one or two adjacent prostate biopsy cores (transrectal ultrasound guided, sextant). In addition we included a group of patients with incidental Tla cancers (diagnosed after prostatectomy, nonpalpable, with less than $5 \%$ tumor in specimen). The proportion of those cancers over the total of tumors diagnosed in our health area was calculated. Also, clinical characteristics of such cancers were recorded (age, PSA, Gleason grade and score), and also therapy given. In cases that underwent RP, pathological findings were also recorded. Finally, survival analysis (Kaplan-Meier) was carried out to describe the natural history of these patients in terms of time to progression and time to death from PCa.

Results: From 819 patients diagnosed of PCa, 46 (5.6\%) presented with focal cancer and 23 (2.8\%) with stage Tla tumors. None of the patients with incidental cancer (Tla) underwent RP opposed to 17 of 46 focal Tlc cancers (37\%). Although none of these cases showed extracapsular extension, seminal vesicle invasion, or lymph node invasion, relevant disease (stage pT2b or higher) was found in 15 cases (88.2\%) and pathological Gleason score $>7$ in 2 cases $(2.9 \%)$. With a mean follow-up time of 37.6 months (standard error 4.26), the probability of being free from any progression was, for Tla cancers at 2 and 5 years, of $75.4 \%$ and $57.1 \%$ respectively, and $94.4 \%$ and $94.4 \%$ respectively for Tlc cases. No PCa deaths were recorded in the presented cases during the mentioned follow-up period.

Conclusion: In our experience, the finding of microscopic or focal cancers in sextant prostate biopsy is related to a high proportion of clinically relevant tumors in RP specimens (88\%). We think that expectant management of patients with such findings in prostate biopsy should be questioned. 
$\mathrm{M}$ otivados por la generalización de la práctica del screening del cáncer de próstata $(\mathrm{CaP})$ durante los últimos años, hemos observado un cambio radical en las características de los tumores detectados. En la actualidad es menos habitual la detección de cánceres en estadios avanzados, y se ha incrementado el diagnóstico de tumores en estadio localizado y no palpables ${ }^{1}$ Asimismo, cada vez es más frecuente el hallazgo de un CaP microscópico o focal tras una biopsia prostática.

Uno de los principales motivos de controversia acerca del beneficio del screening es el fenómeno de la sobredetección, o diagnóstico de tumores sin repercusión clínica. Stamey et al. ${ }^{2}$ establecieron una relación entre el volumen tumoral y su relevancia clínica. Según estos autores, se han considerado tumores clínicamente insignificantes aquellos con volumen menor de 0,5 cc (menor de 0,2 cc para Epstein et al. ${ }^{3}$ ) y grado de Gleason inferior a 4 .

No obstante, es complicado establecer la relevancia clínica del hallazgo de cáncer focal basándonos únicamente en los hallazgos de la biopsia prostática $(<3 \mathrm{~mm}$ en uno o dos cilindros adyacentes). En el presente estudio hemos pretendido calcular la proporción de dichos tumores en nuestro ámbito, investigar su relación con los hallazgos en la pieza de prostatectomía radical (PR), así como establecer su relevancia clínica en términos de progresión y supervivencia.

\section{MATERIAL Y MÉTODOS}

De modo retrospectivo, y partiendo de nuestra base de datos de 819 pacientes con CaP (diagnosticados hasta el 31 de Diciembre de 2004), hemos seleccionado los pacientes con cáncer focal, definiendo éste como un máximo de $3 \mathrm{~mm}$ en uno o dos cilindros adyacentes en la biopsia prostática ${ }^{4}$. Todas las biopsias realizadas en nuestro Centro son dirigidas mediante ecografia transrectal (ECOTR), de modo randomizado sextante. Adicionalmente, hemos incluido un grupo de pacientes con cáncer incidental diagnosticados tras prostatectomía (resección transuretral o adenomectomía) en estadio Tla (no palpables, con tumor en menos del $5 \%$ del tejido obtenido) ${ }^{5}$. Se han excluido los casos con tumores palpables o con evidencia de enfermedad extraprostática o diseminada en estudios de imagen (ECOTR, tomografía axial computadorizada o gammagrafía ósea). En ninguno de los casos con tumor estadio Tla fue realizada biopsia prostática posterior.

Debido al carácter microscópico de los tumores estudiados, en muchos casos no ha sido posible asignar un score o puntuación de Gleason a la muestra obtenida. Por este motivo, en los casos en los que sólo se detectó un foco y fue posible asignar el grado de Gleason, la puntuación de Gleason se obtuvo multiplicando el score por 2 con objeto de mantener la homogeneidad de la muestra para el análisis estadístico.

Se ha calculado la "prevalencia" de cáncer focal en nuestra área sanitaria, en términos de proporción sobre el total de tumores detectados. Posteriormente se han analizado las características clínicas de dichos pacientes, registrando las siguientes variables al diagnóstico: edad, PSA, grado y score de Gleason, así como tratamiento efectuado. En los casos sometidos a PR, se han analizado los parámetros patológicos de la pieza. Aunque no disponemos del volumen tumoral exacto de las piezas, fue posible en todos los casos asignar un estadio patológico concreto en función del tamaño tumoral con respecto al volumen del lóbulo prostático afectado, así como en función de la extensión extracapsular, de la existencia de invasión de vesículas seminales o de la presencia de ganglios invadidos.

Por último, se ha realizado un análisis de supervivencia (Kaplan-Meier) para describir la evolución de estos pacientes en cuanto al tiempo hasta la progresión de la enfermedad y al fallecimiento por $\mathrm{CaP}^{6}$. Debido a que el tratamiento administrado ha sido diferente (y, por tanto, el criterio para definir la progresión de PSA), y teniendo en cuenta que disponemos de un tamaño muestral limitado, hemos considerado el evento progresión como el primero en ocurrir de los siguientes 1) progresión de PSA (PSA sérico $>0,2 \mathrm{ng} / \mathrm{ml}$ tras la PR $\mathrm{y}$ tres elevaciones consecutivas separadas por un periodo mínimo de 6 meses en el resto de los casos), 2) progresión clínica objetiva (progresión en el estadio TNM, o bien cuadro clínico relacionado con la misma, como hematuria por invasión vesical, retención urinaria, atrapamiento ureteral, etc). Para dicho análisis se excluyeron los pacientes con un tiempo de seguimiento inferior a 3 meses. 
El estadiaje tumoral (tanto clínico como patológico) realizado para el presente trabajo se llevó a cabo mediante la clasificación TNM del año $2002^{5}$. $\mathrm{El}$ procesamiento de datos se realizó en un PC con ayuda de los paquetes Microsoft Access y SPSS.

\section{RESULTADOS}

Partiendo de nuestra base de datos con 819 pacientes con $\mathrm{CaP}$, se han seleccionado un total de 69. De ellos, 46 (5,6\%) presentaron cáncer focal y $23(2,8 \%)$ tumores estadio Tla. En la Tabla 1 se resumen las características clínicas al diagnóstico de dichos pacientes, así como el tratamiento realizado.

Ninguno de los pacientes con CaP incidental (T1a) fue sometido a PR, frente a 17 de los 46 cánceres focales T1c (37\%). En la Tabla 2 se ofrecen las características de la pieza de PR en los casos en los que dicha cirugía fue realizada. Cabe destacar que, aunque ninguno de los casos mostró enfermedad extracapsular, invasión de vesículas seminales, o invasión ganglionar, se detectó enfermedad relevante (estadio pT2b o superior) en 15 casos $(88,2 \%)$ y un score de Gleason $>7$ en 2 casos $(2,9 \%)$.

Un total de 60 pacientes (18 incidentales Tla y 42 focales T1c) presentaron un tiempo de seguimiento total evaluable para el análisis Kaplan-Meier de la progresión de la enfermedad. Dicho tiempo de seguimiento osciló entre los 3,7 y los 133,4 meses, con una media de 37,6 y un error estándar de 4,26. El tiempo medio actuarial hasta la aparición de cualquier tipo de progresión fue de 86 y 102,7 meses para los tumores Tla y T1c respectivamente, siendo la mediana de dicho tiempo de 131,4 y 83 meses respectivamente (Tabla 3). La probabilidad de permanecer libre de

Tabla 1. Características en el momento del diagnóstico de los tumores estudiados.

\begin{tabular}{|c|c|c|c|c|}
\hline & & aracterística & óstico & \\
\hline & & Todos & Focal (T1c) & Incidental (T1a) \\
\hline & $\mathbf{N}$ & 69 & 46 & 23 \\
\hline Edad (años) & Mín-Máx & $50-89$ & $50-80$ & $56-89$ \\
\hline & Media (EE) & $66,5(0,95)$ & $65,1(1,04)$ & $69,2(1,85)$ \\
\hline PSA (ng/ml) & Mín-Máx Mediana & $0,8-25,5$ & $1,2-25,5$ & $0,8-22,0$ \\
\hline & & 5,1 & 5,9 & 2,6 \\
\hline Score de Gleason (biopsia) & No determinado & $17(24,7 \%)$ & $12(26,1 \%)$ & $5(21,7 \%)$ \\
\hline & $2-4$ & $21(30,4 \%)$ & $10(21,7 \%)$ & $11(47,8 \%)$ \\
\hline & $5-6$ & $27(39,1 \%)$ & $21(45,7 \%)$ & $6(26,1 \%)$ \\
\hline & 7 & $4(5,8 \%)$ & $3(6,5 \%)$ & $1(4,3 \%)$ \\
\hline & $8-10$ & - & - & - \\
\hline Tratamiento inicial & Observación & $43(62,3 \%)$ & 20 & 23 \\
\hline & Prostatectomía radical & $17(24,6 \%)$ & 17 & 0 \\
\hline & Radioterapia & $9(13,1 \%)$ & 9 & 0 \\
\hline
\end{tabular}

Tabla 2. Datos de la pieza de prostatectomía radical en los 17 pacientes sometidos a dicha intervención.

\begin{tabular}{|c|c|c|c|}
\hline & \multicolumn{3}{|c|}{ Características de la pieza de prostatectomia radical } \\
\hline & & Focal (T1c) & Incidental (T1a) \\
\hline & $\mathbf{N}$ & n (\%) & - \\
\hline Enfermedad extracapsular & & 0 & - \\
\hline Enfermedad relevante $(>=$ pT2b) & & $15(88,2)$ & \\
\hline Penetración capsular & & $2(2,9)$ & - \\
\hline \multirow[t]{4}{*}{ Score de Gleason (pieza) } & $2-4$ & $1(1,5)$ & - \\
\hline & $5-6$ & $12(17,4)$ & - \\
\hline & 7 & $2(2,9)$ & - \\
\hline & $8-10$ & $2(2,9)$ & - \\
\hline Márgen positivo (no uretral) & & $4(5,8)$ & - \\
\hline Invasión vesículas seminales & & 0 & - \\
\hline Invasión ganglionar & & 0 & - \\
\hline
\end{tabular}


Tabla 3. Tiempo actuarial hasta la progresión de cualquier tipo en los pacientes estudiados.

\begin{tabular}{|c|c|c|c|c|c|c|c|c|}
\hline \multirow[b]{3}{*}{ Estadio } & \multicolumn{4}{|c|}{ Media } & \multicolumn{4}{|c|}{ Mediana } \\
\hline & \multirow[b]{2}{*}{ Estimación } & \multirow[b]{2}{*}{$\begin{array}{l}\text { Error } \\
\text { típico }\end{array}$} & \multicolumn{2}{|c|}{$\begin{array}{c}\text { Intervalo de } \\
\text { confianza al } 95 \%\end{array}$} & \multirow[b]{2}{*}{ Estimación } & \multirow[b]{2}{*}{$\begin{array}{l}\text { Error } \\
\text { típico }\end{array}$} & \multicolumn{2}{|c|}{$\begin{array}{l}\text { Intervalo de } \\
\text { confianza al } 95 \%\end{array}$} \\
\hline & & & $\begin{array}{l}\text { Limite } \\
\text { inferior }\end{array}$ & $\begin{array}{l}\text { Limite } \\
\text { superior }\end{array}$ & & & $\begin{array}{l}\text { Limite } \\
\text { inferior }\end{array}$ & $\begin{array}{l}\text { Limite } \\
\text { superior }\end{array}$ \\
\hline Tla (incidental) & 86,0 & 15,6 & 55,4 & 116,6 & 131,4 & 0 & - & - \\
\hline T1c (focal) & 102 & 17,2 & 69,0 & 136,3 & 83,3 & - & - & - \\
\hline Global & 100,9 & 9,6 & 82,1 & 119,6 & 131,4 & 35,5 & 61,8 & 201,0 \\
\hline
\end{tabular}

cualquier tipo de progresión para los Tla a los 2 y 5 años fue de 75,4\% y 57,1\% respectivamente, siendo de $94,4 \%$ y $94,4 \%$ respectivamente para los T1c (Fig. 1).

Finalmente, no se llevó a cabo estudio de mortalidad cáncer-específica, ya que no existieron en esta serie fallecimientos por $\mathrm{CaP}$ hasta la fecha de redacción del presente artículo.

\section{DISCUSION}

El hallazgo principal del presente trabajo radica en la escasa capacidad predictiva (en términos de volumen tumoral) de la biopsia sextante empleada en nuestro entorno. En nuestra experiencia, hasta un $88 \%$ de los cánceres focales no palpables presentaron un estadio patológico $\mathrm{T} 2 \mathrm{~b}$ (tumor que ocupa más de la mitad de un lóbulo prostático) o T2c (presente en ambos lóbulos). Dicho de otro modo, este régimen de biopsia no permite establecer si estamos frente a un tumor insignificante o no. Zackrisson et al., en un estudio similar empleando también biopsia sextante, encontraron que dos tercios de los casos con cáncer focal presentaron un volumen tumoral superior a 0,5 $\mathrm{cc}^{4}$. De modo similar, empleando biopsias de 6-10 cilindros, Boccon-Gibod et al. comprobaron que sólo 30\% de los tumores focales en la biopsia fueron catalogados como insignificantes tras la $\mathrm{PR}^{7}$. Por ello tenemos que concluir que intentar predecir el tamaño tumoral basándonos en biopsias sextantes resulta aventurado. Ante este problema cabrian dos estrategias diferentes: en primer lugar, el aumento del número de cilindros de la biopsia prostática (actitud que, hasta

\begin{tabular}{|c|c|c|c|c|c|c|}
\hline \multicolumn{7}{|c|}{$\begin{array}{l}\text { Figura 1a. Probabilidad de permanecer libre de cualquier progresión. } \\
\text { Pacientes con cáncer de próstata incidental (T1a). Evaluables } n=18 \text {. }\end{array}$} \\
\hline & \multicolumn{6}{|c|}{ Momento de inicio del intervalo (meses) } \\
\hline & 0 & 12 & 24 & 36 & 48 & 60 \\
\hline $\begin{array}{l}\text { Pacientes al comienzo del } \\
\text { intervalo }\end{array}$ & 18 & 16 & 11 & 7 & 5 & 5 \\
\hline Número de eventos & 1 & 3 & 1 & 1 & 0 & 0 \\
\hline $\begin{array}{l}\text { Probabilidad acumulada de no } \\
\text { progresar al final del intervalo }\end{array}$ & 943 & ,754 & .675 &, 571 &, 571 &, 571 \\
\hline \multicolumn{7}{|c|}{$\begin{array}{l}\text { Figura 1b. Probabilidad de permanecer libre de cualquier progresión. } \\
\text { Pacientes con cáncer de próstata focal (T1c). Evaluables } n=42 \text {. }\end{array}$} \\
\hline & \multicolumn{6}{|c|}{ Momento de inicio del intervalo (meses) } \\
\hline & 0 & 12 & 24 & 36 & 48 & 60 \\
\hline $\begin{array}{l}\text { Pacientes al comienzo del } \\
\text { intervalo }\end{array}$ & 42 & 27 & 16 & 11 & 7 & 5 \\
\hline Número de eventos & 2 & 0 & 0 & 0 & 0 & 0 \\
\hline $\begin{array}{l}\text { Probabilidad acumulada de no } \\
\text { progresar al final del intervalo }\end{array}$ & ,944 & ,944 & ,944 & 944 & ,944 & ,944 \\
\hline
\end{tabular}

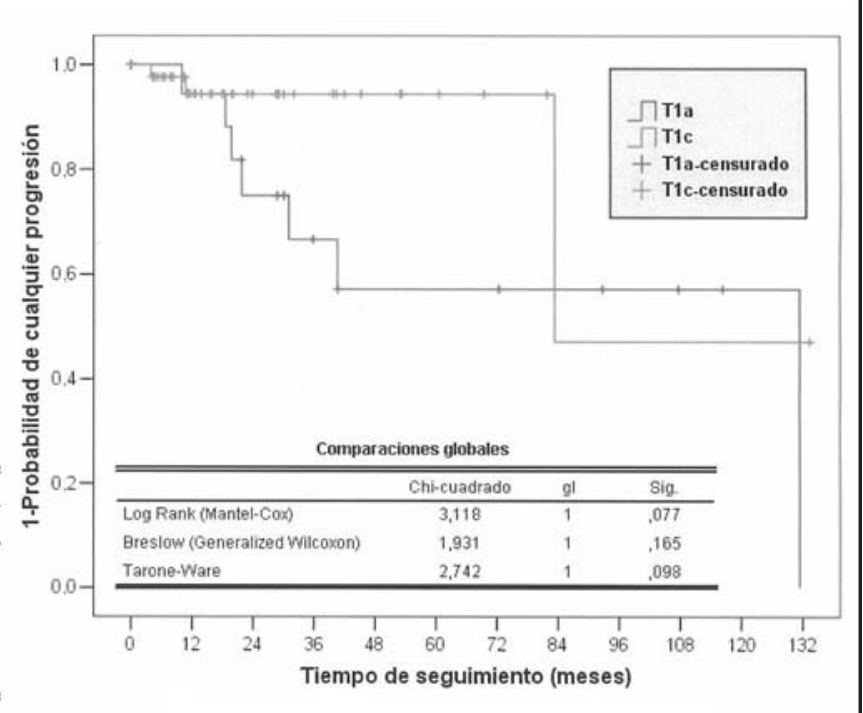

FIGURA 1: Curvas de supervivencia que muestran la probabilidad acumulada de permanecer libre de progresión de cualquier tipo en función del tiempo de seguimiento. A pesar de una tendencia a una mayor progresión en el grupo de pacientes con tumores $\mathrm{Tla}$, la diferencia con el resto de pacientes (T1C) no alcanzó nivel de significación estadistica. 
la fecha, no ha demostrado beneficios en términos de supervivencia sobre la biopsia sextante) ${ }^{8}$ $\mathrm{y}$, en segundo lugar, el ofrecer tratamiento con intención curativa independientemente del carácter focal del tumor en la biopsia sextante. Esta última actitud es la que empleamos en la actualidad en nuestro Centro, ya que no existe un método fiable para categorizar a estos pacientes basándonos únicamente en criterios preoperatorios $^{7}$. Estos resultados contrastan con el 78,6\% de tumores $<0,5$ cc encontrados tras la PR en pacientes con $\mathrm{CaP}$ focal en la biopsia prostática sextante según Postma et al. ${ }^{9}$

Una posible crítica al presente trabajo es el escaso número de pacientes con cáncer focal no palpable (T1c) sometidos a PR (37\%). Aunque la observación sin tratamiento fue la opción terapéutica elegida por el resto de pacientes (en función de su edad, comorbilidad, o preferencia personal), es muy posible que algunos de ellos presentaran un tumor con volumen clínicamente relevante si fueran operados. Quizá, a la vista de estos resultados, sería adecuado hacer más énfasis en una opción terapéutica más agresiva en pacientes candidatos a ello.

Otra limitación del presente estudio es el de un tiempo de seguimiento insuficiente para determinar la evolución de los pacientes en términos de progresión y supervivencia. Por ello, los resultados que hacen alusión a estos dos aspectos deben ser tomados como preliminares.

Por último, hemos incluido cánceres Tla en el presente estudio, con objeto de verificar si su evolución (en términos de progresión) resulta equiparable a la de los tumores focales T1c. Somos conscientes de que algunos de los tumores Tla pueden no compartir la definición de cáncer focal empleada con el resto de tumores. Además, ninguno de ellos fue sometido a biopsia prostática posterior ni a PR. Aunque existió una tendencia a una progresión más temprana de la enfermedad en los Tla, esta diferencia no alcanzó rango significativo. Por este hecho, por la juventud de la serie, además del caracter no homogéneo de las dos poblaciones comparadas, no podemos extraer más conclusiones a este respecto.

\section{CONCLUSIONES}

En nuestro entorno, el hallazgo de cancer microscópico o focal en la biopsia randomizada sextante no se corresponde con las características anatomopatológicas de dicho tumor en la pieza de $\mathrm{PR}$, ya que al menos un $88 \%$ serían catalogados como clínicamente relevantes. Por este motivo, la actitud expectante ante dichos hallazgos en la biopsia prostática debe ser sometida a crítica.

\section{Agradecimientos y soporte financiado}

Financiado en parte por becas del Fondo de Investigación Sanitaria (FIS) (93/0903, 96/0248, 96/1800, 99/0245 y 02/0732).

Nuestro Centro es miembro participante en el "European Randomized Study of Screening for Prostate Cancer (ERPSC)".

A todos los miembros del Servicio de Urología del Hospital de Getafe, tanto en la época actual como en anteriores, que contribuyeron al mantenimiento de nuestra base de datos de pacientes uro-oncológicos.

\section{REFERENCIAS}

1. Luján Galán M, Páez Borda A, Chiva Robles V, Santonja Garriga C, Romero Cajigal I, Berenguer Sánchez A. Tendencias epidemiológicas en cáncer de próstata durante los últimos años. Arch Esp Urol 2004;57(8):817-825.

2. Stamey TA, Freiha FS, McNeal JE, Redwine EA, Whittemore AS, Schmid HP. Localized prostate cancer. Relationship of tumor volume to clinical significance for treatment of prostate cancer. Cancer 1993;71(3 Suppl):933-938.

3. Epstein JI, Walsh PC, Carmichael M, Brendler CB. Pathologic and clinical findings to predict tumor extent of nonpalpable (Stage T1c) prostate cancer. JAMA. 1994;271(5):368-74..

4. Zackrisson B, Aus G, Bergdahl S, Lilja H, Lodding P, Pihl CG, Hugosson J. The risk of finding focal cancer (less than $3 \mathrm{~mm}$ ) remains high on re-biopsy of patients with persistently increased prostate specific antigen but the clinical significance is questionable. J Urol 2004;171:1500-1503.

5. International Union Against Cancer (UICC). TNM Classification of Malignant Tumours, 6th Edition. Sobin LH, Wittekind Ch (Editors). Ed. Wiley. 2002.

6. Kaplan EL, Meier P. Nonparametric estimation from incomplete observations. J Am Stat Assoc. 1958; 53:457-458.

7. Boccon-Gibod LM, Dumonceau O, Toublanc M, Ravery V, Boccon-Gibod LA. Micro-focal prostate cancer: a comparison of biopsy and radical prostatectomy specimen features. Eur Urol. 2005;48(6):895-899.

8. Ciatto S, Zappa M, Villers A, Paez A, Otto SJ, Auvinen A. Contamination by opportunistic screening in the European Randomized Study of Prostate Cancer Screening. BJU Int 2003; 92 Suppl 2:97-100.

9. Postma R, de Vries SH, Roobol MJ, Wildhagen MF, Schröder FH, van der Kwast TH. Incidence and follow-up of patients with focal prostate carcinoma in 2 screening rounds after an interval of 4 years. Cancer 2005;103(4):708-716.

Correspondencia autor: Dr. M. Luján Galán

Servicio de Urología. Hospital Universitario de Getafe.

Ctra. Toledo km 12,500. 28905 Getafe (Madrid)

Tel.: 916833271

E-mail: marcoslujan@terra.es

Información artículo: Cáncer de próstata

Trabajo recibido: enero 2006

Trabajo aceptado: abril 2007 


\section{COMENTARIO EDITORIAL}

1. El análisis estadístico de este artículo es muy adecuado, sin embargo, la selección de los casos y diferentes tratamientos instaurados hacen poco probable extraer conclusión alguna. Quizá sería mejor hacer una selección y análisis pormenorizado de los pacientes sometidos a PR.

2. Un detalle poco claro se puede identificar un estadio T1c con un PSA de 1,2.

\section{Fdo.: J. Morote Robles}

\section{RESPUESTA DE LOS AUTORES}

1. Coincidimos plenamente con el revisor, que ha sabido encontrar las limitaciones de nuestro estudio. Uno de los "puntos flacos" del trabajo es que se intentan analizar los tumores incidentales (Tla) con los focales (T1c). Somos conscientes de que algunos de los tumores Tla pueden no compartir la definición de cáncer focal empleada con el resto de tumores, por eso consideramos que, a la hora de interpretar los resultados, este hecho debe ser tenido en cuenta. Nos ha parecido interesante incluirlos, pero hemos sido cuidadosos de no incluir mención de ello en el apartado "conclusiones".
Por otro lado, el análisis pormenorizado de los pacientes según el tratamiento instaurado haría inviable el análisis debido a la excesiva fragmentación de una muestra con un número limitado de pacientes. Sería muy interesante en una serie más numerosa pero, por lo antes mencionado, hemos creído que lo más honrado era no llevarlo a cabo (el escaso número de pacientes por brazo haría igualmente imposible extraer conclusiones).

2. Creemos adivinar lo que el revisor quiere decirnos con este comentario. Imaginamos que le sorprende que dispongamos de tumores con nivel de PSA $1,2 \mathrm{ng} / \mathrm{ml}$ no palpables. No se trata de un error. Durante el año 2002, dentro del contexto de un programa de screening, hemos sometido a biopsia varones con PSA entre 1 y 3 $\mathrm{ng} / \mathrm{ml}$ con ratio PSA libre/total $<20 \%$. Y, por supuesto, también hemos detectado cánceres en este rango ${ }^{1}$.

\section{REFERENCIAS}

1. Lujan M, Paez A, Miravalles E, Fernandez I, Llanes L, Berenguer A. Prostate cancer detection is also relevant in low prostate specific antigen ranges. Eur Urol 2004;45:155-159.

Fdo.: Los autores 